\title{
Suitability of Synthetic Diamond Films for X-ray Dosimetry Applications
}

\author{
S. P. Lansley*†, G. T. Betzel*, F. Baluti* ${ }^{\star}$, J. Lydon ${ }^{*}$, and L. Reinisch* \\ *Department of Physics and Astronomy \\ University of Canterbury, Christchurch, New Zealand \\ †Email: stuart.lansley@canterbury.ac.nz \\ ${ }^{\ddagger}$ Oncology Service, Christchurch Hospital, Christchurch, New Zealand
}

\begin{abstract}
Simple sandwich-type device structures have been fabricated by deposition of metal contacts on opposing faces of polycrystalline diamond films synthesised using chemical vapour deposition. The electrical characteristics of these devices have been investigated during exposure to a 6 megavolt photon beam from a clinical linear accelerator. The photocurrent appears to vary as the dose rate to the power of $0.78-0.85$. The angular dependence of the photocurrent is less than 10 per cent. Further study on a range of CVD diamond substrates from different manufacturers is on-going.
\end{abstract}

Keywords-diamond; chemical vapour deposition; x-ray; dosimetry; linear accelerator (linac)

\section{INTRODUCTION}

Radiotherapy relies on an optimal radiation dose being applied to a select volume, ultimately just the cancerous volume, of a patient. This, in turn, depends on reliable, sensitive and accurate detectors and instrumentation in order to accurately calibrate the radiotherapy sources and/or directly measure the dose given to a patient.

Diamond is a promising material for use in x-ray dosimetry applications, for reasons such as its near-tissue equivalence (being composed of carbon atoms), radiation hardness, chemical inertness and it is non-toxic. An x-ray detector based on natural diamond is commercially available [1]. However, the use of natural diamond suffers from the scarcity of high quality material with suitable properties, resulting in a high cost and long delivery times.

Synthetic diamond, grown using chemical vapour deposition (CVD), has the potential to eliminate such problems, as it is cheaper and its properties are more reproducible; properties may even be tailored by doping during growth. Numerous research groups have reported their results of x-ray detectors based on CVD diamond, a selection of these can be found in [1,3-13].

\section{EXPERIMENTAL METHODS}

\section{A. Material}

Free-standing polycrystalline chemical vapour deposition (CVD) diamond films were purchased from Diamonex (a division of Morgan Advanced Ceramics), Pennsylvania,
USA [14]. The films were 5 millimetre $\times 5$ millimetre tiles with quoted thicknesses of 100 and 200 micrometres.

The Raman spectrum of a 200 -micrometre-thick film is shown in Fig. 1; the sample was excited using a 514.5nanometre-wavelength argon laser. A sharp diamond Raman peak is clearly evident above a signal background increasing with Raman shift. A couple of small peaks can be seen around 500 and 600 wavenumbers; we have observed these peaks in other films from this manufacturer and in polycrystalline films from other manufacturers.

\section{B. Device Fabrication}

A sandwich-type device structure was fabricated by depositing a 2-millimeter-diameter electrical contact on each side of the diamond film. Silver ( 200 nanometres) was deposited using thermal evaporation through a shadow mask.

The devices were attached to triaxial bulkhead connectors using wires and conductive silver epoxy; the wires acted as both physical support and electrical connections. They were then housed within Perspex enclosures, as shown in Fig. 2. These enclosures were 100 millimetres long and 30 millimetres in diameter, with a 10-millimetre-diameter cavity housing the device. The 10-millimeter-thick wall of the enclosure provides material for the build-up of the $\mathrm{x}$-ray dose; for a 6 megavolt $\mathrm{x}$ ray beam the maximum dose $\left(D_{\max }\right)$ is obtained at a depth $\left(\mathrm{d}_{\max }\right)$ of 15 millimetres [15], but 10 millimetres of build-up material yields a dose of about 98 per cent of the maximum dose. The cavity housing the device contained an air ambient.

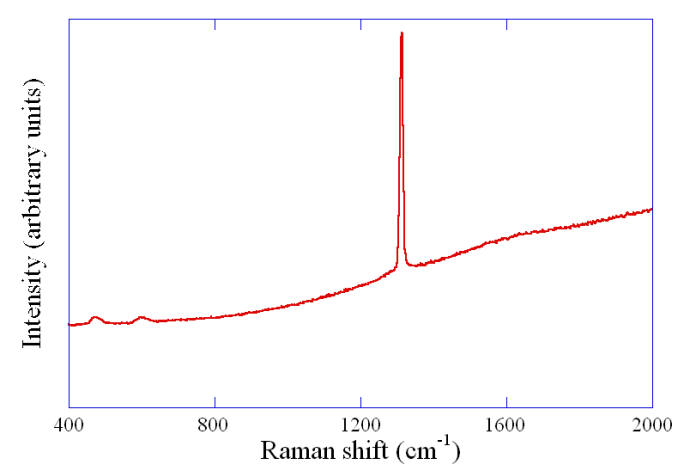

Figure 1. Raman spectrum of a 200-micrometre-thick diamond film; the sample was excited with a 514.5 nanometer Ar line. 


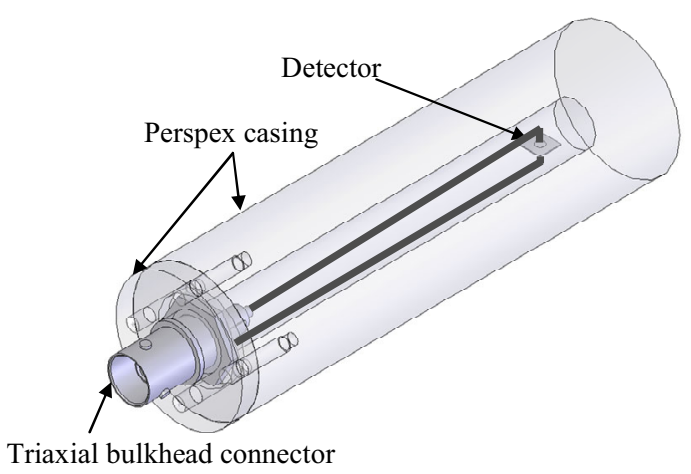

Figure 2. Schematic of the device enclosure.

\section{Measurements}

Current-voltage measurements were obtained using a Keithley 6430 sub-femtoampere remote sourcemeter interfaced to a PC using an IEEE-488.2 USB-to-GPIB interface adapter and a LabView virtual instrument programme.

All x-ray detection measurements derive from exposure to 6 megavolt photon beams from a Varian 600C treatment linear accelerator in the Oncology Service at Christchurch Hospital, New Zealand. The device enclosure was aligned with the gantry axis of rotation and positioned at the linear accelerator isocentre, enabling rotation of the gantry arm around the device whilst retaining constant source-device distance and 10 millimetres of Perspex build-up material between the x-ray source and the device. The device was held in a horizontal orientation with the (positive) bias applied to the uppermost contact, the contact through which the $\mathrm{x}$-rays enter the device when the gantry angle is set to $0^{\circ}$. A 10 centimetre $\times 10$ centimetre field size was used for all measurements.

Triaxial cabling provided electrical connection through the wall of the treatment room to instruments outside the treatment room. A Farmer 2570/1 dosimeter was used to apply a bias to the device and to measure the output of the device. Data were read-out manually as charge (nanocoulombs) measured by the dosimeter over a user-specified time interval, i.e. a time integral of the current through the device. The maximum bias possible from the dosimeter ( $\sim 250$ volts) was used for all devices, yielding an applied electrical field of $\sim 1.25$ volts per micrometre in 200-micrometre-thick material and $\sim 2.50$ volts per micrometre in 100-micrometre-thick material. A bias of half the maximum bias ( $\sim 125$ volts) was also used for the 100 micrometre-thick material so that the same applied field could be used as for the 200-micrometre-thick material.

\section{RESULTS AND DISCUSSION}

All results are from three devices; two using 200micrometre-thick diamond films (devices D01 and D02) and one using a 100-micrometre-thick diamond film (device D03).

\section{A. Device Conductivity Before Irradiation}

Fig. 3(a) shows the current-voltage characteristics of the devices before being irradiated with 6 megavolt x-rays. It appears that device D03 is more conductive than the other two devices. However, when the current is view as a function of

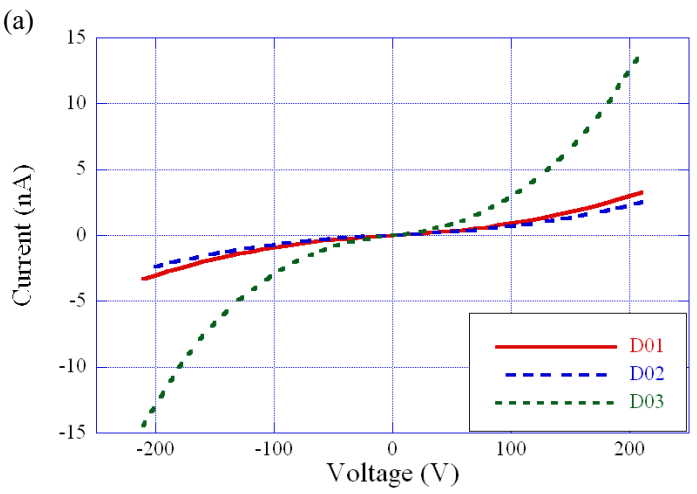

(b)

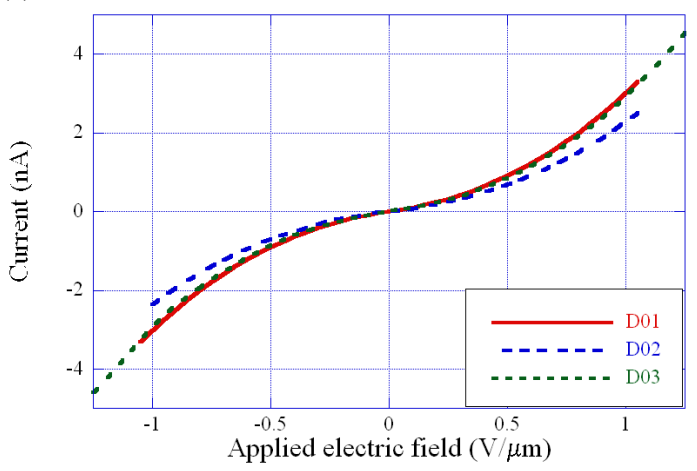

Figure 3. Current versus (a) applied bias and (b) applied electric field for devices D01, D02, and D03 before x-ray irradiation.

the applied electric field, i.e. applied voltage divided by the thickness of the detector material, (Fig. 3(b)) all three devices exhibit similar current-applied electric field characteristics.

\section{B. X-Ray Irradiation}

The integrated charge divided by the integration time (i.e. an approximation of the current through the device) can be seen as a function of time in Fig. 4 for all devices (D01, D02, and D03) at $\sim 250$ volt bias (Fig. 4(a), (b), and (c) respectively) and for device D03 at $\sim 125$ volt bias (Fig. 4(d)). For each device, three groups of five traces can be seen. These correspond to five different dose rates $(50,100,150,200$, and 250 monitor units (MU) per minute) at three different gantry angles $\left(0^{\circ}, 90^{\circ}\right.$, and $\left.180^{\circ}\right)$, as indicated in Fig. 4(a); at $0^{\circ}$ irradiation is from above (i.e. face-on through the positivelybiased contact), at $90^{\circ}$ irradiation is from the side (i.e. edgeon), and at $180^{\circ}$ irradiation is from below (i.e. face-on through the negatively-biased contact).

The signal baseline seen in Fig. 4 corresponds to the $\mathrm{x}$-ray beam being off. It is evident that this baseline is decreasing with irradiation. The baseline appears to reduce exponentially a function of the cumulative dose (Fig. 5); exponential decay curve fits are shown through the data.

Subtraction of the baseline curve fits (Fig. 5) from Fig. 4 yields photocurrent data. A typical set of photocurrent data as a function of the dose rate is shown in Fig. 6; these data were measured on device D01 at a gantry angle of $90^{\circ}$. The data appear to follow an exponential decay characteristic, as shown by the curve fits through the data in Fig. 6. 
7(a) D01@250V

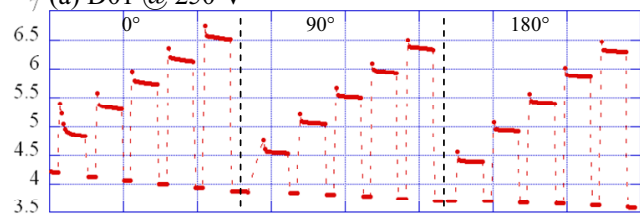

(b) D02@250 V

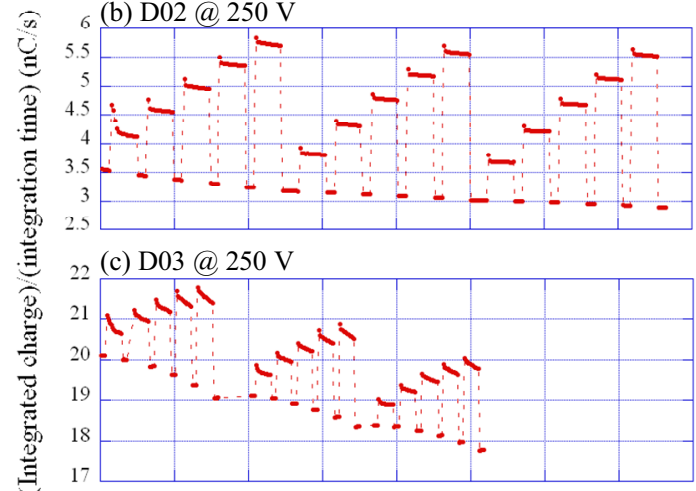

5.5 (d) D03@125 V

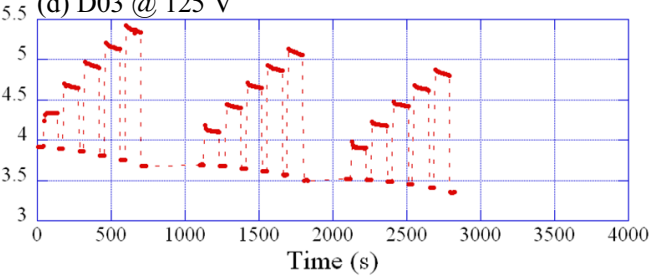

Figure 4. Integrated charge divided by integration time as a function of time for (a) D01 at $\sim 250 \mathrm{~V}$, (b) D02 at $\sim 250 \mathrm{~V}$, (c) D03 at $\sim 250 \mathrm{~V}$, and (d) D03 at $\sim 125 \mathrm{~V}$. Each group of five traces is for dose rates of 50,100, 150, 200, and 250 monitor units (MU) per minute respectively. The left hand group of five traces was taken with the gantry angle set to $0^{\circ}$, the middle group at $90^{\circ}$, and the right group at $180^{\circ}$; as shown in (a).

Fowler states that 'the relationship between photoconductivity $(\sigma)$ and dose rate $(D)$ for insulators is of the form

$$
\sigma \propto D^{\Delta}
$$

where usually $0.5 \leq \Delta \leq 1.0$ ' and that 'if there are no traps or when the excitation rate is so high that traps are unimportant, $\Delta=0.5$ while uniform or quasi-uniform trap distributions over a range of depths in the forbidden energy gap should lead to $\Delta \approx 1$ ' [16]. All devices were kept at constant

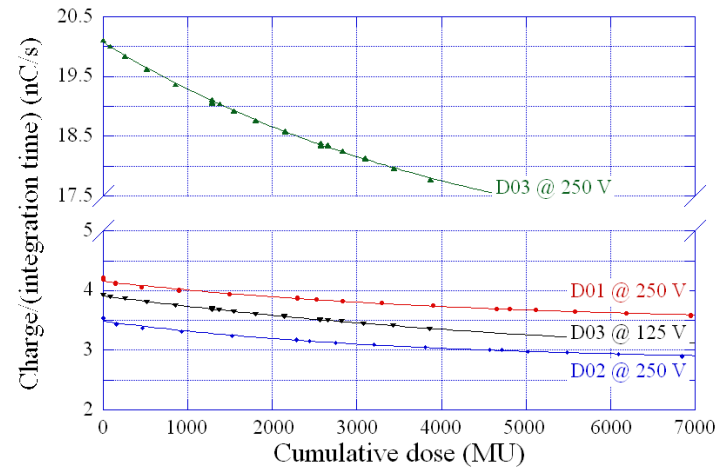

Figure 5. Signal baseline (i.e. dark current) as a function of cumulative dose; exponential decay curve fits are shown through the data.

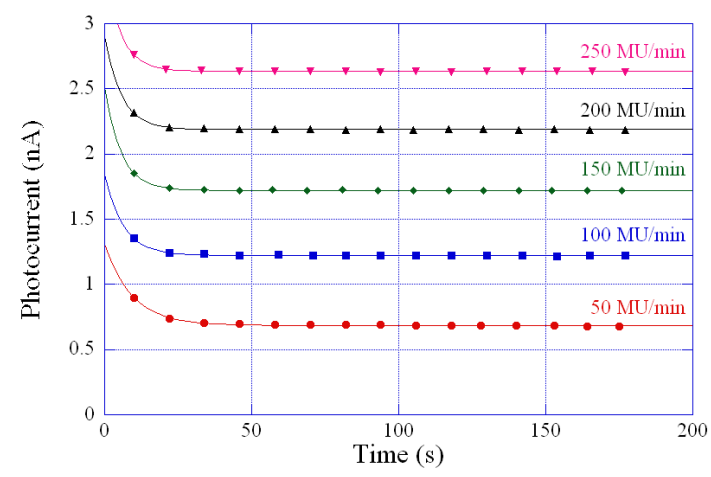

Figure 6. A typical set of photocurrent (i.e. integrated charge divided by integration time, after subtraction of baseline fit) data; this data was measured on device D01 with a gantry angle of $90^{\circ}$. Exponential decay curve fits are shown through the data.

bias, hence the photocurrent $\left(I_{p h}\right)$ is proportional to the photoconductivity $(\sigma)$, so

$$
I_{p h} \propto D^{\Delta}
$$

Fig. 7 shows the steady-state photocurrent plotted against dose rate for device D01; curve fits according to (2) are plotted through the data. Exponent values $(\Delta)$ for all devices, bias values and gantry angles are shown in Table I.

TABLE I. Photocurrent EXPONENT VAlues ( $\Delta$ ) ACCORDing to (2)

\begin{tabular}{|c|c|c|c|c|}
\hline \multirow{2}{*}{ Device } & \multirow{2}{*}{ Bias } & \multicolumn{3}{|c|}{ Gantry angle } \\
\cline { 3 - 5 } & & $\boldsymbol{0}^{\circ}$ & $\mathbf{9 0}^{\circ}$ & $\mathbf{1 8 0}^{\circ}$ \\
\hline D01 & $\sim 250 \mathrm{~V}$ & 0.822 & 0.837 & 0.841 \\
\hline D02 & $\sim 250 \mathrm{~V}$ & 0.839 & 0.850 & 0.846 \\
\hline \multirow{2}{*}{ D03 } & $\sim 250 \mathrm{~V}$ & 0.810 & 0.804 & 0.780 \\
\cline { 2 - 5 } & $\sim 125 \mathrm{~V}$ & 0.820 & 0.798 & 0.790 \\
\hline
\end{tabular}

Fig. 8 shows the normalised photocurrent as a function of gantry angle for device D01 at two dose rates. The variation in photocurrent is fairly small. However, a reduction of about 10 per cent is seen at $135^{\circ}$; this may be due to the $\mathrm{x}$-ray beam being obstructed in some way due to the experimental set-up.

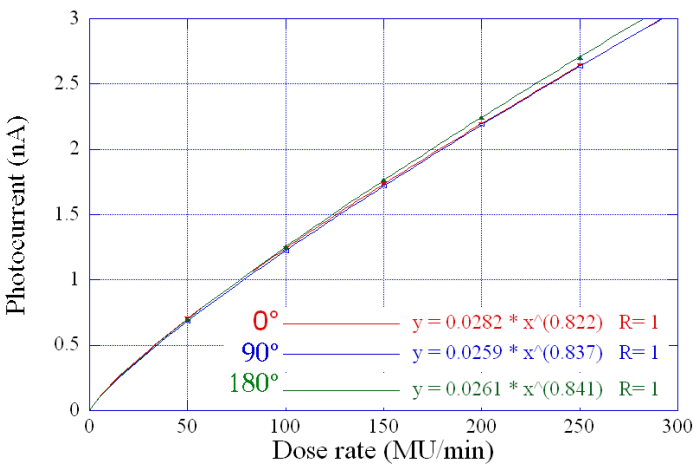

Figure 7. Steady-state photocurrent versus dose rate for device D01; power law curve fits are shown. 


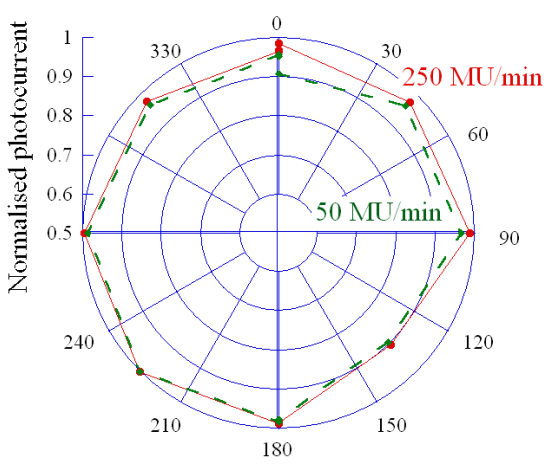

Figure 8. Normalised photocurrent versus gantry angle for device D01 at 50 (dashed line) and 250 (solid line) monitor units per minute.

The Beer-Lambert law states that the intensity of light $(I)$ in a material at some distance $(x)$ from the surface illuminated with incident intensity $I_{0}$ falls off exponentially with $x$, according to some attenuation coefficient $(\alpha)$. Therefore, the amount of light absorbed $\left(I_{a b s}\right)$ in a slab of material of thickness $t$ should be

$$
I_{a b s}=I_{0} \cdot\left(1-\mathrm{e}^{-\alpha t}\right)
$$

Photocurrent is plotted as a function of device thickness and dose rate in Fig. 9; data from all devices at an applied field of $\sim 1.25$ volts per micrometre are shown. Also plotted are curve fits of the form of (3).

\section{Device Conductivity After Irradiation}

The current-applied-electric-field characteristics of the three devices were also measured after x-ray irradiation. The two 200-micrometre-thick devices (D01 and D02) show a reduction in current of 16-17 per cent, whereas the current through the 100-micrometre-thick device (D03) has been reduced by 27 per cent. Device D01 has been exposed to a total of about 11000 monitor units (equivalent to about 140 gray), D02 to about 6900 monitor units ( $\sim 90$ gray), and D03 to about 7700 monitor units ( $\sim 100$ gray); a dose of about 1.3 gray per monitor unit was measured using an ionisation chamber with 10 millimetres of build-up material.

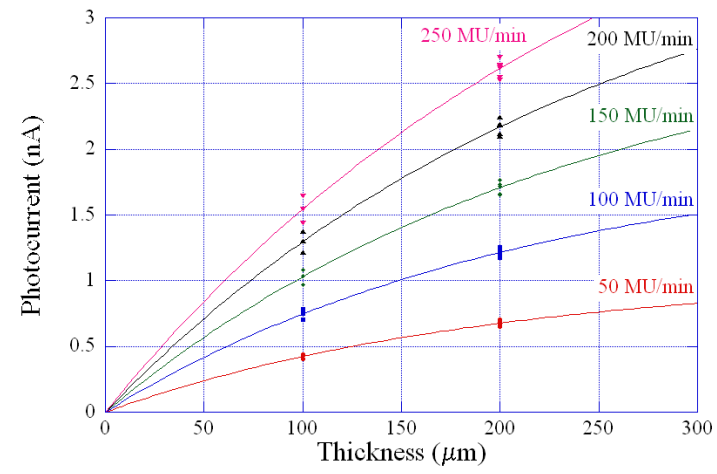

Figure 9. Steady-state photocurrent versus thickness for different dose rates; all data were from the devices when biased at an applied electric field of $\sim 1.25 \mathrm{~V} / \mu \mathrm{m}$, i.e. biased at $\sim 250 \mathrm{~V}$ for devices D01 and D02, and at $\sim 125 \mathrm{~V}$ for device D03. The lines through the data show Beer-Lambert law-type curve fits.

\section{CONCLUSIONS}

Sandwich-type $\mathrm{x}$-ray detectors have been fabricated on Diamonex polycrystalline CVD diamond films. These devices can detect $6 \mathrm{MV}$ x-rays from a clinical linear accelerator. The dark current of these devices reduces exponentially with cumulative dose exposure. Subtraction of this dark current baseline yields a power law relationship between the photocurrent and the x-ray dose rate; the exponent values fit in the range described by Fowler [16]. Only a small variation in photocurrent (a few per cent) is seen at most incident angles.

Further study of detectors on a range of CVD diamond substrates from different manufacturers is on-going.

\section{ACKNOWLEDGMENTS}

SPL thanks the Foundation for Research, Science and Technology (FRST), New Zealand, for a NZ Science and Technology Postdoctoral Fellowship (UOCX0702).

Thanks to Joon Koo Choi for the Raman spectroscopy.

\section{REFERENCES}

[1] "PTW: Diamond Detector"; http://www.ptw.de/diamond_detector0.html

[2] P. Ascarelli et al., "CVD diamond dosimetric response evaluated by Xray absorbers method,” Diam. Rel. Mater., vol. 12, Jul. 2003, pp. 691-5.

[3] M. Assiamah, T. Nam, and R. Keddy, "A synthetic diamond probe for low-energy X-ray dose measurements,” Appl. Rad. Isotopes, vol. 65, May. 2007, pp. 545-552.

[4] P. Bergonzo, D. Tromson, and C. Mer, "Radiation detection devices made from CVD diamond," Semicond. Sci. Technol., vol. 18, Mar. 2003, pp. S105-S112.

[5] M. Bruzzi et al., "CVD diamond particle detectors used as on-line dosimeters in clinical radiotherapy," Nucl. Instrum. Methods A, vol. 518, Feb. 2004, pp. 421-422.

[6] C. De Angelis et al., "Improvements in CVD diamond properties for radiotherapy dosimetry," Rad. Prot. Dosimetry, vol. 120, Sep. 2006, pp. $38-42$.

[7] C. Descamps et al., "Synthetic diamond devices for radio-oncology applications," Phys. Stat. Sol. (a), vol. 203, 2006, pp. 3161-3166.

[8] B. Gorka et al., "Design and characterization of a tissue-equivalent CVD-diamond detector for clinical dosimetry in high-energy photon beams," Physica Medica, In Press, Corrected Proof.

[9] M. Guerrero et al., "Recent improvements on the use of CVD diamond ionisation chambers for radiotherapy applications," Diam. Rel. Mater., vol. 15, 2006, pp. 811-814.

[10] B. Marczewska et al., "Studies on the application of CVD diamonds as active detectors of ionising radiation," Physica B: Condensed Matter, vol. 308-310, Dec. 2001, pp. 1213-1216.

[11] S. Pini et al., "High-bandgap semiconductor dosimeters for radiotherapy applications," Nucl. Instrum. Methods A, vol. 514, Nov. 2003, pp. 135140 .

[12] S. Ramkumar et al., "An assessment of radiotherapy dosimeters based on CVD grown diamond," Nucl. Instrum. Methods A, vol. 460, Mar. 2001, pp. 401-411.

[13] Zhang Minglong et al., "Response of chemical vapor deposition diamond detectors to X-ray," Solid State Comm., vol. 130, May. 2004, pp. $425-428$.

[14] "Diamonex - A Leading Supplier of DLC Coatings and CVD Diamond Products"; http://www.diamonex.com/.

[15] P. Metcalfe, T. Kron, and P. Hoban, The Physics of Radiotherapy XRays from Linear Accelerators, Madison, Wis: Medical Physics Pub, 1997.

[16] J. Fowler, Radiation Dosimetry, New York: Academic, 1966. 